TRANSACTIONS OF THE

AMERICAN MATHEMATICAL SOCIETY

Volume 357 , Number 10, Pages 4215-4223

S 0002-9947(04)03606-2

Article electronically published on November 4, 2004

\title{
SAARI'S CONJECTURE FOR THE COLLINEAR $n$-BODY PROBLEM
}

\author{
FLORIN DIACU, ERNESTO PÉREZ-CHAVELA, AND MANUELE SANTOPRETE
}

\begin{abstract}
In 1970 Don Saari conjectured that the only solutions of the Newtonian $n$-body problem that have constant moment of inertia are the relative equilibria. We prove this conjecture in the collinear case for any potential that involves only the mutual distances. Furthermore, in the case of homogeneous potentials, we show that the only collinear and non-zero angular momentum solutions are homographic motions with central configurations.
\end{abstract}

\section{INTRODUCTION}

In a series of papers published in the 1970s, Don Saari investigated the boundedness of solutions in the Newtonian $n$-body problem. In one of the earliest, [11], he proved that if a solution has constant potential for any finite time interval, then it has both constant potential and constant moment of inertia at all times. In the same paper he stated what is now known as Saari's conjecture: Every solution of the Newtonian n-body problem that has constant moment of inertia is a relative equilibrium.

During a recent visit at the University of Victoria, Don told us that in the 1970s he had found a simple proof of his conjecture in the 3-body case. Unfortunately he never published it, lost his notes, and could not remember the details. Although many people tried to solve his conjecture, nobody has yet succeeded. In the late 1970s and early 1980s, Julian Palmore published two papers, 8 9, in which he claimed to have a proof, but his arguments do not survive a careful scrutiny. In October 2002, Chris McCord gave a talk at the Midwest Dynamical Systems Conference held at the University of Cincinnati, where he presented a proof in the case of three bodies of equal masses, 6]. Also, Rick Moeckel has announced a computerassisted proof for the Newtonian 3-body case. At the time this article was written, their papers had not yet been published.

Recently the interest in Saari's conjecture has grown even more due to the variational proof given by Alain Chenciner and Richard Montgomery for the existence of the figure eight solution, numerically discovered by Cristopher Moore, [2], in 1993. Carles Simó studied this orbit numerically, 12], and found out that its moment of inertia is very close to being a constant all along the motion. This fact prompted Chenciner to ask for analytical arguments that the figure eight solution has no constant moment of inertia.

Received by the editors September 26, 2003 and, in revised form, December 18, 2003. 2000 Mathematics Subject Classification. Primary 70F10; Secondary 70 F07. 
In this paper we make some modest steps towards understanding Saari's conjecture. In particular we prove that the conjecture is true in the collinear case for any number of bodies and for any potential that involves only the mutual distances. This generalizes a result obtained by Pizzetti in 1904, [10, 14, for the Newtonian 3-body problem. Our result is not only more general but the proof we provide is much simpler than the one of the Italian mathematician. Our presentation is organized as follows.

In Section 2 we introduce the equations of motion and several definitions. In Section 3 we solve the collinear case, i.e., the one in which the bodies are on a line that rotates in the plane of motion. We first prove that every non-zero angular momentum and collinear solution of the $n$-body problem given by any potential that depends only on the mutual distances alone, is homographic. This implies that if the moment of inertia is constant, the only solutions of this type are the relative equilibria. Moreover, we show that the only non-zero angular momentum and collinear solutions of the $n$-body problem given by a homogeneous potential of degree $\alpha \neq-2$ are the homographic motions with central configurations. We end our paper with Section 4, in which we give a geometrical interpretation of Saari's Conjecture in the Newtonian case.

\section{EquATIONS OF MOTION}

Consider the planar motion of $n$ interacting bodies $P_{1}, \ldots, P_{n}$. Let the mass and position (with respect to the center of mass) of the the body $P_{i}$ be given by $m_{i}$ and $\mathbf{r}_{i}=\left(x_{i}, y_{i}\right)$. Let $r_{i j}=\left|\mathbf{r}_{i}-\mathbf{r}_{j}\right|$ be the distance between the $i$ th and the $j$ th bodies and denote $x=\left(\mathbf{r}_{1}, \ldots, \mathbf{r}_{n}\right) \in \mathbb{R}^{2 n}$ and $\dot{x}=\left(\dot{\mathbf{r}}_{1}, \ldots, \dot{\mathbf{r}}_{n}\right) \in \mathbb{R}^{2 n}$. Take $L=T-U$ as the Lagrangian function of the system under discussion, where $U$ and $T$ are the potential and the kinetic energy. Then the Euler-Lagrange equations for the body $P_{i}$ are

$$
m_{i} \ddot{\mathbf{r}}_{i}=-\frac{\partial U}{\partial \mathbf{r}_{i}}
$$

With the scalar product

$$
\langle x, \dot{x}\rangle=\sum_{i=1}^{n} m_{i} \mathbf{r}_{i} \cdot \dot{\mathbf{r}}_{i}
$$

and the notation

$$
\nabla=\left(m_{1}^{-1} \nabla_{1}, \ldots, m_{n}^{-1} \nabla_{n}\right)
$$

the equations of motion take the simpler form

$$
\dot{x}=y, \quad \dot{y}=-\nabla U,
$$

where $\nabla_{i}$ is the $i$ th gradient component.

The moment of inertia and the kinetic energy can be written as

$$
I=\langle x, x\rangle, \quad T=\frac{1}{2}\langle y, y\rangle .
$$

A central configuration of the $n$-body problem is a configuration $x \in \mathbb{R}^{2 n}$ that satisfies the algebraic equations

$$
\nabla U(x)=\omega^{2} \nabla I
$$

for some constant $\omega$ (see [7, 13, 14, for more details). 
A given solution $x=x(t)$ of the $n$-body problem is called homographic if, in the barycentric coordinate system, the configuration of the bodies is similar to itself when $t$ varies. By this we mean that there exist a scalar $\nu=\nu(t)>0$, an orthogonal 2-matrix $\Omega=\Omega(t)$ such that for every $i$ and $t$ one has

$$
\mathbf{r}_{i}=\nu(t) \Omega(t) \mathbf{r}_{i}^{0}
$$

where $\mathbf{r}_{i}, \nu, \Omega$ correspond to an arbitrary $t$ and $\mathbf{r}_{i}^{0}$ denotes $\mathbf{r}_{i}$ at some initial instant $t=t_{0}$.

There are two limiting types of homographic solutions. One appears when the configuration is dilating without rotation, i.e., $\Omega(t)$ is the identity matrix. Such solutions are called homothetic. The other shows up when the configuration is rotating without dilatation, i.e., $\nu(t)=1$. These particular homographic solutions are characterized by

$$
\mathbf{r}_{i}=\Omega(t) \mathbf{r}_{i}^{0}
$$

and are called relative equilibria. In such cases the system rotates about the center of mass as a rigid body, the angular velocity is constant and the mutual distances do not changes when $t$ varies. The relative equilibria are the solutions we are interested in, and they make the object of Saari's Conjecture.

In the following sections we will deal with two types of potentials that are more general than the Newtonian one: first, when the potential energy is a function of the mutual distances only and, second, when the potential is a homogeneous function of degree $\alpha$. In the latter case the Lagrangian function is of the form

$$
L=T-U=\frac{1}{2} \sum_{i=1}^{n} m_{i} \dot{\mathbf{r}}_{i}^{2}+\beta \sum_{i>j} m_{i} m_{j}\left|\mathbf{r}_{i}-\mathbf{r}_{j}\right|^{\alpha},
$$

where $\beta=1$ if $\alpha<0$, whereas $\beta=-1$ if $\alpha>0$. The corresponding Hamiltonian function is

$$
H=T+U=\sum_{i=1}^{n} \frac{\mathbf{p}_{i}^{2}}{2 m_{i}}-\beta \sum_{i>j} m_{i} m_{j}\left|\mathbf{r}_{i}-\mathbf{r}_{j}\right|^{\alpha} .
$$

The Lagrangian and Hamiltonian above describe the Newtonian n-body problem when $\alpha=-1$ and $\beta=G$, where $G$ is the gravitational constant.

\section{THE COLLINEAR N-BODY PROBLEM}

In this section we will prove Saari's Conjecture in the collinear case for any potential that depends on the mutual distances alone. By the collinear case we mean the one in which the bodies are on a line that rotates in the plane around the center of mass of the bodies.

Let $\mathbf{K}$ be the total angular momentum and let $\mathbf{K}_{i}$ be the angular momentum of the body $P_{i}$ about the center of mass. Denote by $\mathbf{M}_{i}$ the moment of the forces for the body $P_{i} . \mathbf{F}_{i j}$ is the force acting on the body $P_{i}$ while interacting with $P_{j}$. It is well known (see [14]), and easy to check, that every collinear solution is planar. Assume that the potential $U$ is function of the mutual distances alone. Note that $\mathbf{F}_{i j}=\nabla_{\mathbf{r}_{i j}} U$. In this setting, we can prove the following result.

Theorem 1. Every collinear and non-zero angular momentum solution of the $n$ body problem given by a potential that depends only on the mutual distances is homographic. 
Since $\mathbf{r}_{i}$ and $\mathbf{F}_{i j}$ are collinear,

$$
\dot{\mathbf{K}}_{i}=\mathbf{M}_{i}=\sum_{\substack{j=0 \\ j \neq i}}^{n} m_{i} \mathbf{r}_{i} \times \mathbf{F}_{i j}=0 .
$$

Consequently $\mathbf{K}_{i}$ is a constant vector. If $K_{i}$ is the component of $\mathbf{K}_{i}$ orthogonal to the plane of motion, then $K_{i}=c_{i}$, where $c_{i}$ is a constant. We can also write the component of the angular momentum orthogonal to the plane of motion as

$$
K_{i}=c_{i}=m_{i} r_{i}^{2}(t) \omega(t),
$$

where the angular velocity $\omega$ is the same for all the bodies, since they belong to a line. Let us note that the vectors $r_{i}$ and the angular velocity $\omega$ may depend on time.

Now consider the ratio between the components of the angular momentum of any two bodies $P_{i}$ and $P_{j}$. Using (12), we get

$$
\frac{c_{i}}{c_{j}}=\frac{m_{i}}{m_{j}} \frac{r_{i}^{2}}{r_{j}^{2}}
$$

consequently the ratio between distances is

$$
\frac{r_{i}(t)}{r_{j}(t)}=\sqrt{\frac{m_{j} c_{i}}{m_{i} c_{j}}}
$$

Consequently the geometrical configuration of the $n$ bodies remains similar to itself as $t$ varies. This concludes the proof.

The particular Newtonian case of Theorem 1 was proved by Pizzetti in 1904 for the 3-body case, 10, 14. Unaware of his result, we found the above shorter, simpler and more general proof. Using Theorem 1, we can now confirm Saari's conjecture in the collinear case for any number $n$ of bodies.

Corollary 1. Consider the n-body problem given by a potential that depends on the mutual distances alone. Then the only solutions that are collinear, have non-zero angular momentum, and constant moment of inertia are the relative equilibria.

Proof. The component of the total angular momentum orthogonal to the plane of motion can be written as

$$
K=\sum_{i=0}^{N} K_{i}=\omega \sum_{i=0}^{N} m_{i} r_{i}^{2}=\omega I=C,
$$

where $I$ is the moment of inertia. Since $I$ and $K$ are constant, $\omega$ is constant too. The fact that mutual distances are also constant follows immediately from (12). Hence we showed that if the moment of inertia is constant, the corresponding solutions are relative equilibria. This completes the proof.

Corollary 1 completely characterizes the collinear solutions with constant moment of inertia, but Theorem 1 is not the strongest result that can be proved. Indeed, in 1767 Leonhard Euler showed that if in the Newtonian case three bodies of arbitrary masses are arranged initially on a line, as in Figure 1, such that the ratio $A B / B C$ of their mutual distances has a certain value given by a formula depending on the masses, and if suitable initial velocities are assigned to the particles, then they will move periodically on ellipses remaining on a line at all times. Such motions are called homographic motions with central configurations. 


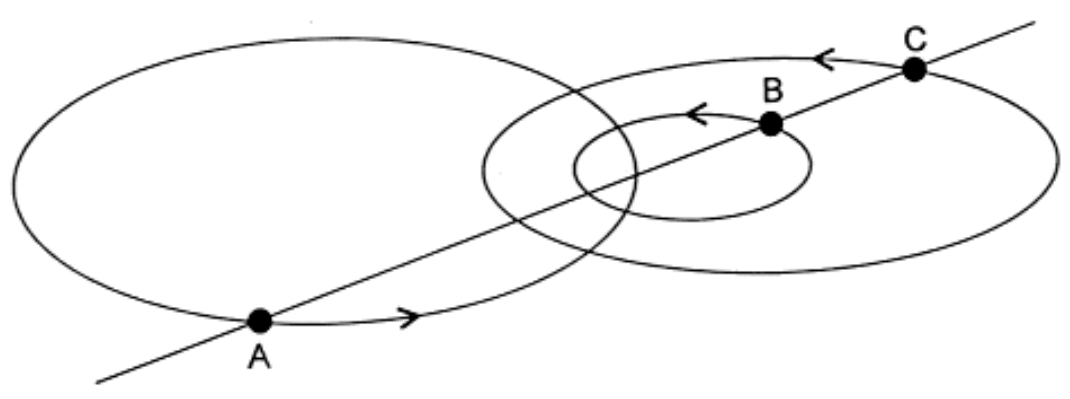

FiguRE 1. The Eulerian solution of the three-body problem.

This leads to a natural question: "Are there any collinear orbits given by homographic solutions with central configurations?" It turns out that the answer is positive if the potential is homogeneous of degree $\alpha \neq-2$. More precisely, we have the following result.

Theorem 2. The only collinear and non-zero angular momentum solutions of the $n$-body problem given by a homogeneous potential of degree $\alpha \neq-2$, are the homographic motions with central configurations. In particular if the moment of inertia is constant, the solutions are relative equilibria.

Proof. The first part of the proof follows immediately from Theorem 1 and Proposition 2.14 in [1, which shows that if the potential is homogeneous of degree $\alpha \neq-2$, then the homographic solutions are homographic with central configurations. The second part follows from Corollary 1. This concludes the proof.

We further present an alternative proof of Theorem 2, which uses Sundman's inequality. Differentiating the expression of the angular momentum of the body $P_{i}$ (see (12)) with respect to the time variable and using (11), we find that

$$
\dot{K}_{i}=2 m_{i} r_{i} \dot{r}_{i} \omega(t)+m_{i} r_{i}^{2} \dot{\omega}(t)=0 .
$$

This implies that

$$
\dot{r}_{i}=-\frac{r_{i}}{2} \frac{\dot{\omega}}{\omega} .
$$

With these preparations, we can rewrite the kinetic energy,

$$
T=\frac{1}{2} \sum_{i=1}^{N}\left(m_{i} \dot{r}_{i}^{2}+m_{i} r_{i}^{2} \omega^{2}\right),
$$

in the form

$$
T=\frac{C}{2}\left(\frac{\dot{\omega}^{2}}{4 \omega^{3}}+\omega\right),
$$

where we replaced $\dot{r}_{i}$ with the expression in (17) and $I$ with $C / \omega$.

Now consider Sundman's inequality

$$
2 T I-J^{2} \geq|C|^{2},
$$


where

$$
J=\sum_{i=1}^{N} m_{i} \mathbf{r}_{i} \cdot \dot{\mathbf{r}}_{i}=\sum_{i=1}^{N} m_{i} r_{i} \dot{r}_{i}
$$

(see [1, 14] for a derivation). Using (17), we can write

$$
J=-\sum_{i=1}^{N} m_{i} r_{i}^{2} \frac{\dot{\omega}}{2 \omega}=-I \frac{\dot{\omega}}{2 \omega}
$$

and since $C=I \omega$, we get

$$
J=-\frac{\dot{\omega}}{2 \omega^{2}} C
$$

With the help of equations (19) and (23), we can write the left-hand side of Sundman's inequality as

$$
2 T I-J^{2}=\frac{C^{2}}{\omega}\left(\frac{\dot{\omega}^{2}}{4 \omega^{3}}+\omega\right)-\left(-\frac{\dot{\omega}^{2} C}{2 \omega^{2}}\right)^{2}=C^{2},
$$

which becomes an equality in this case. Therefore the solutions are homographic (as proved in [1]) and since $U$ is homogeneous of degree $\alpha \neq-2$ ([1], Proposition $2.14)$, they are either solutions with central configurations or rigid motions. In the latter case the moment of inertia $I$ is constant and we can show as in Corollary 1 that the solutions are relative equilibria. This concludes the alternative proof.

Let us remark that the homogeneity of the potential is an essential hypothesis of the theorem since there are quasi-homogeneous potentials (see [4]), in particular the Lennard-Jones potential, which have central configurations that depend on the mutual distances and hence, in those cases, different and more complex types of motions might occur.

\section{A geometrical interpretation}

In this section we will give a geometrical interpretation of the above results in the Newtonian case. Our discussions with Don Saari revealed that he had developed a similar geometric understanding of the problem long before we found ours.

The moment of inertia can be written in terms of the relative distances as

$$
I=\frac{1}{2 M} \sum_{i=1}^{n} \sum_{j=1}^{n} m_{i} m_{j} r_{i j}^{2}
$$

For three collinear bodies, the potential energy and the moment of inertia contain only three terms, so it is natural to try to represent the manifolds $I=$ constant and $U=$ constant in the space of relative distances.

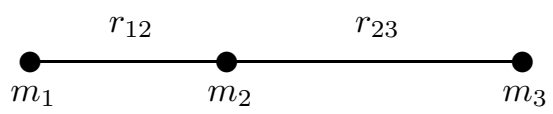

Figure 2. Three bodies on a line for a fixed ordering of the masses.

Moreover since the bodies are on a line, they are subject to additional constraints. For instance if one fixes the ordering of the masses as in Figure 2 the condition is 
that $r_{13}=r_{12}+r_{23}$, which means that the bodies are confined to a plane in the space of relative distances. Since three bodies can be ordered in three ways, the corresponding conditions define three planes in the space of mutual distances, as we illustrate in Figure 3, which depicts the intersection of the manifolds $U=$ constant, $I=$ constant, and the three planes that give the collinear configurations. It is now easy to see that, once ordering the bodies, the collinear relative equilibria correspond to the case in which the intersection of $U=$ constant and $I=$ constant restricted to the plane is just one point.

From the purely geometric point of view there are three possible cases for the outcome of the above intersection: empty, one point, or two points. But by Moulton's theorem we know that for each ordering there is only one relative equilibrium and thus the relative equilibrium must correspond to the case when the intersection of $I=$ constant and $U=$ constant is tangent to the plane (see Figure 31). This is an alternative proof of Saari's Conjecture in the collinear case of three bodies.

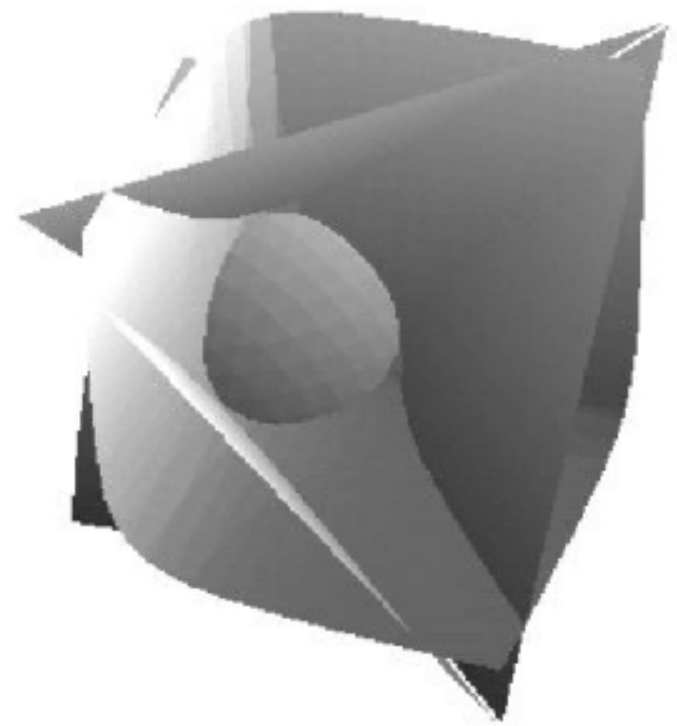

Figure 3. Intersection of the manifolds $U=$ constant, $I=$ constant with the three planes in the case of equal masses.

However, this proof cannot be generalized to $n>3$. Indeed, the geometry becomes more complicated as the number of bodies increases. It is first easy to find the following recurrence relation for the number $M(n)$ of the mutual distances of $n$ bodies:

$$
M(n+1)=M(n)+n .
$$

Since $M(3)=3$, this recurrence relation can be solved; it leads to the formula

$$
M(n)=\frac{n(n-1)}{2} .
$$

The number of linear relations that must be verified so that the $n$ bodies are on a line can also be found. The recurrence relation that gives the number $R(n)$ of 
linear relations is

$$
R(n+1)=2 R(n)-R(n-1)+1 .
$$

The initial conditions $R(2)=0$ and $R(3)=1$ lead to the formula

$$
R(n)=\frac{n(n-1)}{2}-(n-1) .
$$

This means that we have $M(n)$ unknowns, $R(n)$ linear relations, and two more relations given by $U=$ constant and $I=$ constant. Consequently if $n>3$ the number of mutual distances is larger than the number of relations. In general this implies that the problem could have infinitely many solutions, unless the intersection is degenerate, in which case the intersection reduces to one point. As we have shown in the previous section, Saari's Conjecture is true in the general case, so the intersection is indeed one point. For $n>3$, however, it is impossible to draw this conclusion only from geometrical considerations. But it is interesting to remark that the dynamics of the problem leads to this unlikely geometric configuration.

\section{ACKNOWLEDGMENTS}

We are indebted to Don Saari for the many discussions he had with us about his conjecture in September 2002 while he was a PIMS Distinguished Chair at the University of Victoria. Without Don's insight, enthusiasm, and energy, we would have never finalized this research. We would also like to acknowledge our sponsors: Manuele Santoprete was supported by a University of Victoria Fellowship while completing his Ph.D. degree with Florin Diacu at the University of Victoria, Florin Diacu received partial support from the NSERC of Canada Grant OGP0122045 and from a Research Fellow Grant of the Pacific Institute for the Mathematical Sciences, while Ernesto Pérez-Chavela was supported in part by Proyecto CONACYT of México.

\section{NOTE ADDED IN PROOF}

At a conference held at the beginning of June 2004 in Cala Gonone, Sardinia, the first author gave a talk on Saari's Conjecture. At the end of the talk, Rick Moeckel asked what happens in the rectilinear case (when all the bodies move on a fixed line). The author answered that collisions always occur, so the potential becomes infinite and the moment of inertia cannot be constant, therefore the conjecture is false. But he could not remember the collision proof on the spot. Rick Moeckel found an alternative argument the next day, using McGehee coordinates. The proof the first author thought about can be found on page 45 of

F. Diacu, Singularities of the $N$-body Problem, in Classical and Celestial Mechanics -The Recife Lectures, pp. 35-62, H. Cabral and F. Diacu, editors, Princeton Univ. Press, Princeton, NJ, 2002.

\section{REFERENCES}

[1] A. Albouy and A. Chenciner, Le problème des $n$ corps et les distances mutuelles, Inventiones Mathematicae 131, 151-184 (1998). MR98m:70017

[2] A. Chenciner and R. Montgomery, A remarkable periodic solution of the three body problem in the case of equal massess, Annals of Mathematics 152, 881-901 (2000). MR 2001k:70010

[3] F. Diacu, Singularities of the N-Body Problem-An Introduction to Celestial Mechanics, Editions CRM, Montreal, 1992. MR94b:70015 
[4] F. Diacu, Near-collision dynamics for particle systems with quasihomogeneous potentials, Journ. Differential Equations 128, 58-77 (1996). MR97i:70018

[5] J.E. Marsden, Lectures on Mechanics, Cambridge University Press: Cambridge (1992). MR $93 \mathrm{f}: 58078$

[6] C. McCord, Saari's conjecture for the three-body problem with equal massess, preprint.

[7] K. Meyer and G,R. Hall, Introduction to Hamiltonian Dynamical Systems and the N-Body Problem, Applied Mathematical Sciences, 90, Springer: New York (1992). MR 93b:70002

[8] J. I. Palmore, Relative equilibria and the virial theorem, Celestial Mechanics 19, 167-171 (1979). MR80i:70014

[9] J. I. Palmore, Saari's conjecture revisited, Celestial Mechanics 25, 79-80 (1981). MR.83c:70011

[10] P. Pizzetti, Casi particolari del problema dei tre corpi, Rendiconti della Reale Accademia dei Lincei s.5 v. 13, 17-26 (1904).

[11] D. Saari, On bounded solutions of the n-body problem, Periodic Orbits, Stability and resonances, G.E.O., Giacaglia (Ed.), D. Riedel, Dordrecht, 76-81 (1970). MR42:8753

[12] C. Simó, New families of Solutions in $N$-Body Problems, Congress of Mathematics, Vol. I (Barcelona, 2000), 101-115, Progr. Math., 201, Birkhäuser, Basel (2001). MR.2003g:70012

[13] S. Smale, Topology and mechanics, II, The planar n-body problem, Inventiones Mathematicae, 11, 45-64 (1970). MR 47:9671

[14] A. Wintner, The Analytical Foundations of Celestial Mechanics, Princeton University Press (1941). MR3:215b

Pacific Institute for the Mathematical Sciences and Department of Mathematics and Statistics, University of Victoria, P.O. Box 3045 STN CSC, Victoria, British Columbia, Canada V8W 3P4

E-mail address: diacu@math.uvic.ca

Departamento de Matemáticas, Universidad Autónoma Metropolitana-Iztapalapa, Apdo. 55534, MÉxico, D.F., MÉxico

E-mail address: epc@xanum.uam.mx

Department of Mathematics, University of California, Irvine, 294 Multipurpose Science \& Technology Building, Irvine, California 92697

E-mail address: msantopr@math.uci.edu 